\title{
Factores de riesgo asociados a aborto bovino en la cuenca lechera del departamento de Nariño
}

\section{Risk factors associated with bovine abortion in a dairy region in the department of Nariño}

\author{
Bibiana Benavides B, ${ }^{1}$ Esp, Cesar Jurado, ${ }^{2}$ Esp, Darío Cedeño Q, ${ }^{1 *}$ M.Sc. \\ ${ }^{1}$ Universidad de Nariño, Facultad de Ciencias Pecuarias, Departamento Salud Animal, \\ Pasto, Colombia. ${ }^{2}$ Practica privada. *Correspondencia: dcedeno@udenar.edu.co
}

Recibido: Abril 14 de 2009; Aceptado: Marzo 10 de 2010.

\section{RESUMEN}

Objetivo. Identificar los factores asociados al aumento en la probabilidad de ocurrencia del aborto bovino, permitiendo la implementación de programas de control efectivos. Materiales y métodos. Se trabajó con una prevalencia superior al $5 \%$ según datos obtenidos por Colácteos en el 2003 de la cuenca lechera. Los factores de riesgo asociados a la presentación de aborto se agruparon en agentes etiológicos de origen infeccioso y de origen no infeccioso. Para la identificación de los factores de riesgo se analizó la información para los años 20062007, obtenida de registro que reportan la presentación de abortos en fincas de la cuenca lechera. Los datos insuficientes se complementaron con la aplicación de una encuesta para cada finca que incluyó las variables consideradas como factores de riesgo. El diseño para identificar los factores correspondió a un estudio de casos y controles, donde se calculó el Odds Ratio para cada variable en estudio y se validó con el modelo de regresión logística multivariado. Resultados. Se encontró en las variables, ausencia de sistemas de drenaje con un OR 4.42(P 0.006) y la ausencia de pozo séptico con OR 5.59(P 0.033).Conclusiones. Los abortos se presentan con mayor frecuencia en vacas adultas entre el segundo y cuarto parto, durante el último tercio de la gestación y en los primeros 5 meses del año. Los factores de riesgo asociados a la presentación de aborto fueron la ausencia de sistemas de drenaje y de pozo séptico para el manejo de aguas residuales y aguas lluvias.

Palabras clave: Factores de riesgo, feto abortado, Holstein.

\section{ABSTRACT}

Objective. To identify the risk factors associated with the increasing of the probability of bovine abortion enabling the execution of effective control programs. Materials and methods. Abortion in the department of Nariño has prevailed more tan 5\%, according to the information gathered by Colacteos in 2003 . Risk factors in relation to abortion existence were grouped in 
infectious and non-infectious source. In order to identify the risk factors, the data gathered through files and record systems was analyzed between 2006-2007. This data reported abortion cases in milk-producing farms. The insufficient information was completed by a survey carried out in every farm including the variables which were rated as risk factors. A case and control study was the design used to identify these factors in which the Odds Ratio for every variable was estimated and then validated with the regression logistic multivariate model. Results. The values obtained for the variables were the shortage of drainage systems with an OR 4.42 ( $p$ 0.006) and the shortage of a septic well with OR 5.59 ( $p$ 0.033). Conlusions. the variables considered as risk factors were the shortage of drainage systems and the shortage of a septic well.

Key words: Risk factors, aborted fetus, Holstein

\section{NTRODUCCI ÓN}

El aborto es por definición, la pérdida del producto de la concepción antes de que sea viable, a partir del periodo fetal (aprox. 42 días) hasta antes de los 260 días en caso del bovino (1). El aborto bovino es un factor limitante del desarrollo ganadero por las pérdidas económicas que genera en el sistema productivo. El aborto puede presentarse en forma esporádica, endémica o en forma de brote y puede ser de origen infeccioso o no infeccioso, por lo que resulta difícil establecer cual es el agente causal (2). Algunos estudios indican que agentes infecciosos como la diarrea viral bovina (DVB) y la Neospora caninum son de mayor relevancia en la presentación del aborto en el ganado lechero (3). En una investigación realizada en el departamento de Nariño los agentes infecciosos de mayor prevalencia para el año de 1999 fueron: Brucella abortus, Leptospira hardjo, Campylobacter foetus, Trichomona foetus y rinotraqueitis infecciosa bovina (4).

Los factores de riesgo asociados a la presentación de abortos incluyen características del individuo, nutrición, prácticas de manejo reproductivo, condiciones ambientales (5). La movilización de animales, prácticas de manejo, programas de vacunación, presencia de mascotas y otros animales de corral en los sistemas de producción (6) Para su determinación se debe contar con el adecuado registro de los eventos presentados en las fincas durante el período de estudio.

Por estas razones se deben realizar estudios regionales para identificar la presencia de factores de riesgo reportados para este evento y así determinar los puntos de control en el establecimiento de los programas de prevención y control para los hatos lecheros en Nariño.

El objetivo de este estudio fue identificar los factores asociados al aumento en la ocurrencia del aborto bovino en la cuenca lechera del departamento de Nariño.

\section{MATERI ALES Y MÉTODOS}

Sitio de estudio. El estudio se realizó en fincas ubicadas en el departamento de Nariño en los municipios pertenecientes a la cuenca lechera: Pupiales, Guachucal, Tuquerres y Pasto.

Selección de fincas. Se incluyeron en el estudio las fincas que llevan un sistema de información actualizado por lo cual se tomaron las que se encontraban en la base de datos de Colácteos, que en la cuenca lechera corresponden a 273 fincas, distribuidas de la siguiente forma Guachucal 59, Pasto 84, Tuquerres 76 y Pupiales 54.

Criterios de inclusión. Para determinar las fincas participantes en el estudio se definieron los siguientes criterios de inclusión:

- Fincas con registro de datos que pertenezcan a la cuenca lechera.

- Animales que aborten con permanencia superior a 2 meses en la finca en estudio.

- Se definio aborto como pérdida después 
del día 42 y con expulsión de membranas $\mathrm{y} / \mathrm{o}$ feto.

- Presentación de aborto en los años 20062007.

- Los abortos debian aparecer registrados en el sistema de información.

Los casos fueron fincas con eventos registrados y ocurridos en el período de 2006-2007, de animales que pertenecian a la finca y cumplian con los criterios de inclusión. Los controles eran fincas que no tenian registrado ningún evento en el período 2006-2007 y pertenecian al mismo municipio en el que se encuentran los casos. La tasa de asignación del estudio fue de 1 y a cada caso se le asignó un control dentro del mismo municipio.

Diseño, calculo del tamaño de la muestra y analisis de resultados. El diseño para identificar factores de riesgo correspondió a un estudio de casos y controles, en el cual se calculó el Odds Ratio (OR) para cada variable.

Se calculó el tamaño de muestra para las fincas con base a la información de las proporciones esperadas para cada grupo (caso-control), se conocia el valor de Pc y $\mathrm{Pn}$, se obtuvo la siguiente fórmula: (7).

$\mathrm{n}=\mathrm{n}_{1}(\mathrm{k}+1)$

$$
n 1=\frac{\left(Z_{1-\alpha / 2}+Z_{1-\beta}\right)^{2}}{\{4 k / k+1\}\left\{\sin ^{-1}(\sqrt{P c})-\sin ^{-1}(\sqrt{P n})\right\}^{2}}
$$

Donde:

Error tipo I: 0.05. Error tipo II: 0.1 .

Pc: proporción del factor entre los controles.

Pn: proporción del factor entre los casos.

K: tasa de asignación entre grupos.

Para el estudio se consideró un error tipo I de 0.05 , error tipo II de 0.1 , proporción del factor entre los controles del 30\%, proporción del factor entre los casos $60 \%$. La tasa de asignación entre los grupos: fue de 1 control por un caso, con hipótesis a dos colas.

El tamaño de muestra calculado fue de 112 fincas, es decir 56 casos y 56 controles. Distribuidos de la siguiente forma 24 en Guachucal (40\%), 34 en Pasto (40.4\%), 30 en Tuquerres (39\%) y 28 en Pupiales (52\%), las frecuencias relativas se calcularon con base en el número de fincas que se encontraron en la base de datos de Colácteos.

Las variables en estudio (Tabla 1 ) correspondieron a los factores de riesgo que pueden llevar a la presentación de abortos de acuerdo con las causas potenciales identificadas en la bibliografía de referencia. Algunas variables incluidas en el instrumento de recolección no se analizaron como factores de riesgo, pero a través de tablas de frecuencias son bases para caracterizar las fincas con el evento en estudio (casos).

Tabla 1. Variables del instrumento de recolección y función en el estudio.

\begin{tabular}{ll}
\hline \multicolumn{1}{c}{ VARIABLE } & \multicolumn{1}{c}{ FUNCIÓN } \\
\hline $\begin{array}{l}\text { Información de animales } \\
\text { que abortan }\end{array}$ & Caracterización \\
Tiempo de gestación & \\
Número de partos & \\
Producción de leche & \\
Mes de presentación & \\
Prácticas de Manejo & \\
Vacunación & \\
Tratamientos médicos & \\
Suplementación & \\
Sistema de reproducción & \\
Estratificación del hato & \\
Manejo nocturno & \\
Almacenamiento de alimento & \\
Medio Ambiente & Factores de riesgo \\
Agua de bebida & \\
Drenaje de potreros & \\
Tipo de abono para praderas & \\
Presencia de otros animales & \\
Saneamiento Básico & \\
Presposición de material abortado de Riesgo & \\
Manejo de aguas residuales & \\
\hline
\end{tabular}

\section{RESULTADOS}

La información se obtuvo a través de los registros y la aplicación de la encuesta en cada finca incluida en el estudio. Esta información se codificó para cada variable y 
se construyó una base de datos, la cual permitió como primer paso la realización de una estadística descriptiva basada en las frecuencias de presentación de las variables que se incluyen para describir la dinámica del aborto en las fincas que hacen parte del estudio.

Dentro de estas variables el número de partos permitió una aproximación de la edad y de esta forma la asociación del evento con la etapa reproductiva del animal y con la producción de leche expresada como el promedio de la producción del hato/día (Tabla 2).

Con los datos codificados y tabulados se realizó el cálculo del Odds Ratio para cada factor en estudio, con su respectivo intervalo de confianza y valor de P. Las variables que presentaron más de una categoría se llevaron a la escala nominal dicotómica y se analizaron como variables "Dummy" con el objetivo de definir claramente la ausencia o presencia de cada uno de los factores identificados previamente como factores de riesgo. Estos
Tabla 2. Descripción de la presentación de aborto en la cuenca lechera de Nariño.

\begin{tabular}{lcc}
\hline \multicolumn{1}{c}{ VARIABLES } & n & $\%$ \\
\hline Tiempo de Gestación & & \\
Primer tercio & 6 & 11 \\
Segundo tercio & 18 & 32 \\
Último tercio & 32 & 57 \\
Número de Partos & & \\
Primero & 13 & 23 \\
2-4 partos & 39 & 70 \\
> 4 partos & 4 & 7 \\
Mes de presentación & & \\
Enero-Abril & 22 & 39 \\
Mayo-Julio & 24 & 43 \\
Agosto-Diciembre & 10 & 18 \\
Producción láctea & & \\
$<10$ Its & 9 & 16 \\
10-20 Its & 34 & 61 \\
>20 Its & 13 & 23 \\
\hline
\end{tabular}

Tabla 3. Resultados de Odds Ratio (OR), intervalo de confianza (IC) y significancia (P) para las variables relacionadas con los factores de riesgo.

\begin{tabular}{lccc}
\hline \multicolumn{1}{c}{ VARIABLE } & VALOR OR & IC & VALOR DE P \\
\hline Vacunación CR & 10.96 & $<3.49<\mathrm{OR}<36.67$ & 0.0000008 \\
Suplementación & 0.35 & $<0.11<\mathrm{OR}<1.10$ & 0.043 \\
Almacenamiento & 0.42 & $<0.16<\mathrm{OR}<1.06$ & 0.042 \\
Abono (estiércol) & 0.54 & $<0.22<\mathrm{OR}<1.34$ & 0.146 \\
Prácticas reproductivas & & & \\
Monta directa & 0.82 & $<0.31<\mathrm{OR}<2.15$ & 0.654 \\
Inseminación artificial & 1.08 & $<0.47<\mathrm{OR}<2.44$ & 0.848 \\
Mixto & 1.17 & $<0.50<\mathrm{OR}<2.76$ & 0.689 \\
Estratificación & & & \\
NO & 2.52 & $<0.54<\mathrm{OR}<13.15$ & 0.185 \\
SI & 0.54 & $<0.12<\mathrm{OR}<2.23$ & 0.34 \\
Manejo nocturno & & & \\
Estabulado & 1.22 & $<0.30<\mathrm{OR}<5.01$ & 0.75 \\
Recogido & 6.83 & $<2.77<\mathrm{OR}<17.16$ & 0.0000016 \\
Libre & 0.13 & $<0.05<\mathrm{OR}<0.34$ & 0.0000014 \\
Tipo de agua & & & \\
Nacimiento & 0.55 & $<0.24<\mathrm{OR}<1.27$ & 0.124 \\
Quebrada & 2.47 & $<0.92<\mathrm{OR}<2.77$ & 0.047 \\
Potable & 1.47 & $<0.57<\mathrm{OR}<3.79$ & 0.3827 \\
Ausencia de drenaje & 5.65 & $<2.01<\mathrm{OR}<16.43$ & 0.00016 \\
Presencia de mascotas & 6 & $<1.89<\mathrm{OR}<20.24$ & 0.00041 \\
Otros animales & 2.04 & $<0.14<\mathrm{OR}<58.57$ & 0.55 \\
Roedores & 12.65 & $<4.03<\mathrm{OR}<42.44$ & 0.0000001 \\
Ausencia de pozo séptico & 3.33 & $<1.09<\mathrm{OR}<10.60$ & 0.0137 \\
Letrinas & 1.6 & $<0.63<\mathrm{OR}<4.10$ & 0.28 \\
No Tto. Aguas residuales & 2.04 & $<0.14<\mathrm{OR}<58.57$ & 0.55 \\
\hline
\end{tabular}


factores se analizaron individualmente con el programa Epilnfo 6 que permite obtener la tabla tetracorica con sus respectivos valores de $\mathrm{P}$ e intervalos de confianza

En la tabla 3 se encuentran los valores de OR, obtenidos para cada una de las variables incluidas en el estudio que son reportadas como factores de riesgo para las diferentes causas de aborto.

Las variables que por su OR, intervalo de confianza y valor de $\mathrm{P}$ se identificaron como factor de riesgo fueron recogido nocturno, presencia de mascotas, ausencia de drenaje, ausencia de pozo séptico, vacunaciones y presencia de roedores. Estas variables se analizaron a través del modelo de regresión logística multivariado en el programa estadístico SPSS10, que permite asociar el desarrollo del evento con la presencia o no de estos factores y de esta forma determinar la significancia estadística y definir la validez de la asociación encontrada con el evento en estudio.

De acuerdo con la tabla 4, la predicción del modelo equivale al $78.6 \%$, lo cual significa que de las 56 fincas que reportaron abortos, el modelo lo predijo en 44 fincas.

En la tabla 5 se presentan los resultados obtenidos en el modelo multivariado para los cuales se analiza el valor $\operatorname{Exp}(B)$ equivalente
Tabla 4. Clasificación de la predicción del modelo.

\begin{tabular}{cccc}
\hline Observado & $\mathbf{0}$ & $\mathbf{1}$ & $\begin{array}{c}\text { Porcentaje } \\
\text { correcto }\end{array}$ \\
\hline 0 & 43 & 13 & $76.8 \%$ \\
1 & 12 & 44 & $78.6 \%$ \\
Porcentaje global $49.1 \%$ & $50.9 \%$ & $77.7 \%$ \\
\hline
\end{tabular}

al OR de cada variable. De esta forma se definió cuales fueron los factores de riesgo para el estudio.

Al analizar los resultados del Exp (B) se concluyó que la presentación del evento de aborto aumentó con la ausencia de drenaje (OR 4.2) y pozo séptico (OR 5.8).

\section{SCUSIÓN}

Según los resultados obtenidos en el estudio, los abortos en los municipios de la cuenca lechera del departamento de Nariño se presentan con mayor frecuencia en vacas adultas entre el segundo y cuarto parto, con una producción de leche entre 10 y 20 litros/ día, durante el último tercio de la gestación y en los primeros 5 meses del año.

En la tabla 2, se observa que durante el segundo tercio de gestación se presentó el $32 \%$ de los casos relacionado con agentes de tipo infeccioso como Trichomona foetus,

Tabla 5. Estimaciones de parámetros para el modelo

\begin{tabular}{|c|c|c|c|c|c|c|c|c|}
\hline \multirow[b]{2}{*}{ Definición } & \multirow[b]{2}{*}{ B } & \multirow[b]{2}{*}{ Error } & \multirow[b]{2}{*}{ Wald } & \multirow[b]{2}{*}{ GI } & \multirow{2}{*}{ Sig } & \multirow{2}{*}{$\operatorname{Exp}(\mathrm{B})$} & \multicolumn{2}{|c|}{ Intervalo de confianza al 95\% para exp (B) } \\
\hline & & & & & & & Limite inferior & Limite superior \\
\hline Intersección & 0.677 & 1.512 & 0.201 & 1 & 0.654 & & & \\
\hline Recogido $=0$ & -1.029 & 0.529 & 3.792 & 1 & 0.051 & 0.357 & 0.127 & 1.007 \\
\hline Recogido $=1$ & $0 *$ & & & 0 & & & & \\
\hline Mascota $=0$ & -0.957 & 1.342 & 0.509 & 1 & 0.476 & 0.384 & 0.02766 & 5.331 \\
\hline Mascota $=1$ & $0 *$ & & & 0 & & & & \\
\hline Drenaje $=0$ & 1.442 & 0.529 & 7.424 & 1 & 0.006 & 4.229 & 1.499 & 11.93 \\
\hline Drenaje $=1$ & $0 *$ & & & 0 & & & & \\
\hline Pozosept $=0$ & 1.765 & 0.804 & 4.817 & 1 & 0.028 & 5.844 & 1.208 & 28.27 \\
\hline Pozosept=1 & $0^{*}$ & & & 0 & & & & \\
\hline Vacunas $=0$ & -1.298 & 0.605 & 4.599 & 1 & 0.032 & 0.273 & 0.0834 & 0.894 \\
\hline Vacunas=1 & $0 *$ & & & 0 & & & & \\
\hline Roedores $=0$ & -1.505 & 0.673 & 5.007 & 1 & 0.025 & 0.222 & 0.05939 & 0.83 \\
\hline Roedores=1 & $0 *$ & & & 0 & & & & \\
\hline
\end{tabular}


Neospora caninum e IBR (8). Para el primer tercio de gestación se reportaron eventos causados por IBR que se manifiestan con momificaciones, mortalidades embrionarias, y repeticiones de celo (9), las cuales no son analizadas en este estudio La presentación en el último tercio de la gestación se relacionó directamente con la presencia de agentes de tipo infeccioso como son Brucella, Leptospira y Neospora. Es de aclarar que las causas virales y de origen no infeccioso pueden presentarse durante todo el período de gestación por lo cual el tiempo de gestación no sugiere ninguna asociación con la presentación del aborto (10).

En cuanto a la producción de leche se identificaron un mayor número de casos en las vacas de una producción media (10-20 litros) dentro del hato, sin embargo no se ha podido asociar la producción de leche o grasa a la presentación del aborto, esto concuerda con lo reportado con Chebel et al (11) en el año 2004. Zambrano y Thurmond (12) afirman que estas variables pueden confundirse con la enfermedad que afecta la producción de leche y la viabilidad fetal.

La mayoría de abortos se presentaron en vacas entre el segundo y cuarto parto, lo que lleva a hacer una común asociación de la edad con el número de gestaciones, concluyendo que un mayor número de partos disminuiría la capacidad del animal para mantener la preñez (13), aunque Zambrano y Thurmond (12) en el 2009 en la sabana de Bogotá encontraron que las vacas de mayor edad que no han abortado son retenidas dentro de la explotación. Estudios recientes encontraron que no existe asociación estadística entre el número de partos, la producción de leche, el número de inseminaciones o el protocolo usado con ese fin (11).

Para el estudio no pudo delimitarse la época del año de presentación de los abortos a ninguna estación ya que durante el primer trimestre se espera un clima cálido y seco relacionado con el verano lo cual sería similar a lo encontrado en el Valle de California donde para esta época del año se presenta la edad gestacional de mayor riesgo (3 y 8 meses) (14) y durante el siguiente trimestre se esperan lluvias, lo cual no ha podido asociarse con la presentación de abortos (11).

Dentro del análisis univariable se encontraron diferentes variables que pueden considerarse factores de riesgo de acuerdo a su valor de OR y valor de P (Tabla 3), pero el análisis multivariado no las considera como tal. Sin embargo, se considera que estas pueden ser relevantes en la presentación de abortos y se consideran a continuación.

El recogido en potrero como práctica de manejo nocturno reporta un OR 6,83, $(<2.77<$ OR $<17.16)$ y valor $\mathrm{P} 0,0000016$. Este factor de riesgo lo reporta Radostits (15) para los agentes de tipo infeccioso, ya que el hacinamiento y estrés al que se someten los animales facilita los procesos de transmisión vertical y persistencia de agentes infecciosos como es el caso de Brucella, Leptospira, DVB e IBR. En Nariño la práctica de recoger al ganado en un solo potrero en la noche es altamente difundida, lo cual se atribuye a problemas de seguridad en las zonas rurales y se considera una medida efectiva de para evitar el robo de animales

La presencia de mascotas OR $6(<1.89$ $<O R<20.24)$ y valor $\mathrm{P} 0.00041$ y la presencia de roedores OR $12.65(<4.03<0 R<42.44)$ y valor $\mathrm{P} 0,00000001$, se reportan como factores de riesgo para Neospora, Brucella y Leptospira ya que se convierten en huéspedes, potenciales transmisores y perpetuadores de la infección en la finca, lo cual coincide con lo reportado por Murray (16), Kokabiyik y Cetil (17) y Moreno et al (18). A diferencia de estos estudios las variables mencionadas no fueron definidas como factores de riesgo dentro de este estudio porque todas las fincas reportan presencia de animales diferentes a los bovinos y esta condición no permite definir la diferencia de exposición para casos y controles. Por esta razón se debe implementar otro tipo de estudio epidemiológico que permita encontrar asociación causal para definir estas variables como factores de riesgo (19). Además para el caso de los roedores su presencia depende de la época del año, la utilización agropecuaria de los suelos de la finca y la forma de almacenamiento de los granos y 
concentrados (20), factores que se tuvieron en cuenta dentro del análisis pero que igualmente no presentan diferencia para los casos y controles.

Dentro del estudio se analizaron prácticas que se consideran factores de protección como es el caso de la vacunación y la práctica de inseminación artificial la más difundida en esta zona de Nariño. La variable de vacunación presenta un OR de 10,96 $(<3,49<$ OR $<36,67)$ y valor $\mathrm{P}$ de 0,0000008 , por lo cual se considera que no es un factor protector para la presentación de abortos causados por agentes inmunoprevenibles. Este resultado puede verse influido por la no realización de pruebas diagnósticas para agentes infecciosos como lo recomiendan los programas preventivos para este tipo de enfermedades $(21,22)$.

La inseminación artificial como la práctica reproductiva más difundida reduce la presentación de enfermedades de transmisión venérea como Trichomonas, Brucella y DVB, pero no se considera como único factor de protección para estas entidades como lo comenta Zambrano y Thurmond (12).
De acuerdo con modelo de regresión logística multivariado los factores de riesgo asociados a la presentación de aborto para la cuenca lechera incluyen la ausencia de sistemas de drenaje y de pozo séptico para el manejo de aguas residuales y aguas Iluvias. Lo cual coincide con estudios realizados para agentes como Leptospira y Neospora ya que la ausencia de sistemas de drenaje favorece la formación de cuerpos de agua en especial en épocas de lluvia, facilitando el mantenimiento de estos agentes en el predio $(14,18,20,23,24)$. Sumado a este factor, se encontró el inadecuado manejo de aguas residuales por ausencia de pozo séptico lo que favorece la diseminación de agentes de tipo infeccioso.

En conclusión, los abortos se presentan con mayor frecuencia en vacas adultas entre el segundo y cuarto parto, durante el último tercio de la gestación y en los primeros 5 meses del año. Los factores de riesgo asociados a la presentación de aborto incluyeron la ausencia de sistemas de drenaje y de pozo séptico para el manejo de aguas residuales y aguas lluvias.

\section{REFERENCI AS}

1. Drost M, Jousan F, Hansen P. Factors associated with early and mid-to-late fetal loss in lactating and nonlactating Holstein cattle in a hot climate. Gainesville: Departments of Animal Sciences and Large Animal Clinical Sciences, University of Florida; 2004.

2. Carpenter T, Chriel M, Anderson M, Wulfson $L$, J ensen $A$, Hove $H$, et al. An epidemiologic study of late term abortions in dairy cattle in Denmark, July 2000-August 2003. Prev Vet Med 2006; 77(3-4): 215-229.

3. Björkman C, Alenius S, Manuelsson U, Uggla A. Neospora caninum and Bovine Virus Diarrhoea Virus I nfections in Swedish Dairy Cows in Relation to Abortion. Vet J 2000; 159(2): 201-6
4. González G, Patiño R. Principales agentes infectocontagiosos del aborto e infertilidad en el ganado lechero de Nariño y alto Putumayo. Nariño, Colombia: Ministerio de Agricultura y Desarrollo Rural. Regional No. 5. CORPOICA; 1999.

5. Murray RD. A field investigation of causes of abortion in dairy cattle. Vet Rec 2004; 154:692-693

6. Kirkbride C. Etiologic agents detected in a 10-year study of bovine abortions and stillbirths. J Vet Diagn Invest 1999; 4: 175-180.

7. Hahai H, Khurshid A. A Second Bibliography on the Teaching of Probability and Statistics. J Off Stat 1996, 4(3). 
8. Anderson ML. Infectious causes of bovine abortion during mid- to late-gestation. Theriogenology 2007; 68: 474-486.

9. Betancur C, González M, Reza L. Seroepidemiología de la rinotraqueitis infecciosa bovina en el municipio de Monteria, Colombia. Rev MVZ Córdoba 2006; 11(2): 830-836.

10. Gadicke P, Monti G. Aspectos epidemiológicos y de análisis del síndrome de aborto bovino. Arch Med Vet 2008; 40:223-234.

11. Chebel RC, Santos JE, Reynolds JP, Cerri $\mathrm{RL}$, Juchem SO, Overton M. Factors affecting conception rate after artificial insemination and pregnancy loss in lactating dairy cows. Anim Reprod Sci 2004; 84:239-255.

12. Zambrano J, Thurmond M. Aproximación epidemiológica para medir y entender el aborto bovino. Rev Med Vet Zoot 2009; 56:309-326.

13. Starbuck MJ, Dailey RA, Inskeep EK. Factors affecting retention of early pregnancy in dairy cattle. Anim Reprod Sci 2004; 84:27-39.

14. Thurmond MC, Anderson ML, Blanchard PC. Secular and seasonal trends of Neospora abortion in California dairy cows. J Parasitol 1995; 81: 364-367.

15. Radostits, Otto. Herd Health: Food animal production Medicine. Philadelphi: Saunders Company; 2000.

16. Murray, R.D. A field investigation of causes of abortion in dairy cattle. Vet Rec 2004. 154:692-693.
17. Kokabiyik AL, Cetil C. Bovine leptospirosis in South of Marmara. Region of Turkey. Revue de Medicine Veterinarie. 2004. 12: 606-608.

18. Moreno JF, Rentería TB, Searcy R, Montaño $R$. Seroprevalencia y factores de riesgo asociados a la brucelosis bovina en hatos lecheros de Tijuana, Baja California. Tec Pecu Mex 2002; 40(3): 243-249.

19. Dohoo I, Martin W, Stryhn H. Veterinary Epidemiologic Research. Charlottetown, Canada: Atlantic Veterinary College; 2004.

20. Vanasco N.B, Sequeira G,. Tarabla H.D. Associations between leptospiral infection and seropositivity in rodents and environmental characteristics in Argentina. Prev Vet Med 2003; 60:227-235.

21. Vargas D, Jaime J, Vera V. Perspectivas para el control del virus de la diarrhea viral bovina (DVB). Rev Colomb Cienc Pecu 2009; 22:677-688.

22. Bezek DM. Bovine virus diarrhea virus infection: Individual and herd diagnosis. Compend Contin Educ Vet 1995. 17(8):57-64.

23. Grooms DL. Reproductive losses caused by bovine viral diarrhea virus and leptospirosis. Theriogenology 2006; 66: 624-628.

24. Garcia-Vazquez Z, Rosario-Cruz R,RamosAragon A, Cruz-Vazquez C, Mapes-Sanchez G. Neospora caninum seropositivity and association with abortions in dairy cows in Mexico. Vet Parasitol 2005; 134: 61-65. 